\title{
Genetically Modified Mosquitoes ${ }^{1}$
}

\author{
Eric P. Caragata, Yoosook Lee, and Eva A. Buckner²
}

\section{Introduction}

The goal of projects that release genetically modified (GM) mosquitoes is to control mosquito populations and reduce the risk of mosquito-borne diseases. Much of the controversy surrounding GM mosquitoes can be attributed to lack of information and/or misinformation. The purpose of this publication is to provide science-based information about GM mosquitoes to the public as well as those involved in mosquito control in Florida, the United States, and beyond. Below we provide information on Oxitec's GM mosquito pilot projects in Florida and outline why GM mosquitoes are being investigated as a tool for mosquito control. We also present a series of answers to frequently asked questions about GM mosquitoes including how they are developed and what potential impact they may have on environmental and human health. A list of additional resources is provided at the end of this publication for readers who are interested in further information on this topic.

\section{Oxitec's GM Mosquito Release in the Florida Keys}

Oxitec is a biotechnology company founded in 2002 out of Oxford University in the United Kingdom. This company in partnership along with the Florida Keys Mosquito Control District will carry out small pilot experimental releases as allowed by the US Environmental Protection Agency (EPA) under experimental use permit in the Florida Keys. The pilot projects will involve releasing Oxitec's GM adult,
Aedes aegypti male mosquitoes (strain OX5034) into small, defined areas to test their ability to reduce the population of wild i.e., non-GM Ae. aegypti mosquitoes. These projects, which will be the first time that GM mosquitoes are released into the field in the United States, begin in spring 2021 and will continue through spring 2022.

\section{Aedes aegypti Mosquitoes}

Aedes aegypti (Figure 1), also known as the dengue or yellow fever mosquito, is an annoying, biting pest and the species most responsible for transmitting dengue (DENV), chikungunya (CHIKV), yellow fever (YFV), and Zika (ZIKV) viruses to humans. Aedes aegypti mosquitoes are commonly found in tropical and sub-tropical regions, as well as in some temperate areas. Though all male mosquitoes do not bite, female mosquitoes bite and feed on blood to obtain the nutrients they need to produce their eggs. Female Ae. aegypti mosquitoes preferentially bite humans and therefore, Ae. aegypti mosquitoes typically live where people do and are commonly found in urban and suburban environments. If a female Ae. aegypti mosquito bites someone who is already infected with DENV, CHIKV, YFV, or ZIKV, she becomes infected with the virus and can spread it to the next person she bites if they survive long enough for virus to escape mosquito tissue barriers (Figure 2). Because of this, Ae. aegypti is a major threat to human health in the many areas of the world where the mosquito species and at least one of the viruses it transmits co-occur. Ae. aegypti is an invasive species established in many areas of Florida (including the Florida Keys). Regular introductions

1. This document is ENY-2066, one of a series of the Entomology and Nematology Department, UF/IFAS Extension. Original publication date July 2021. Visit the EDIS website at https://edis.ifas.ufl.edufor the currently supported version of this publication.

2. Eric P. Caragata, assistant professor; Yoosook Lee, assistant professor, and Eva A. Buckner, assistant professor and state Extension specialist; Entomology and Nematology Department, UF/IFAS Florida Medical Entomology Laboratory, Vero Beach, FL 32962.

The Institute of Food and Agricultural Sciences (IFAS) is an Equal Opportunity Institution authorized to provide research, educational information and other services

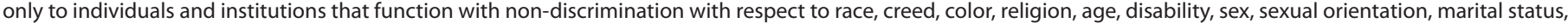

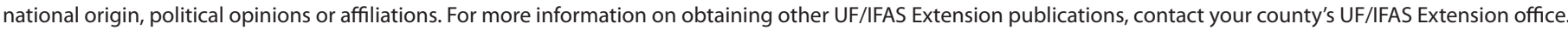
U.S. Department of Agriculture, UF/IFAS Extension Service, University of Florida, IFAS, Florida A \& M University Cooperative Extension Program, and Boards of County Commissioners Cooperating. Nick T. Place, dean for UF/IFAS Extension. 
of DENV, CHIKV, and ZIKV have caused local outbreaks that resulted in 190,12, and 300 human cases, respectively, over the past 15 years in the state with almost all of those cases occurring in southern Florida (Kendrick et al. 2014; Rey 2014; Phillip et al. 2019; FDOH 2021).

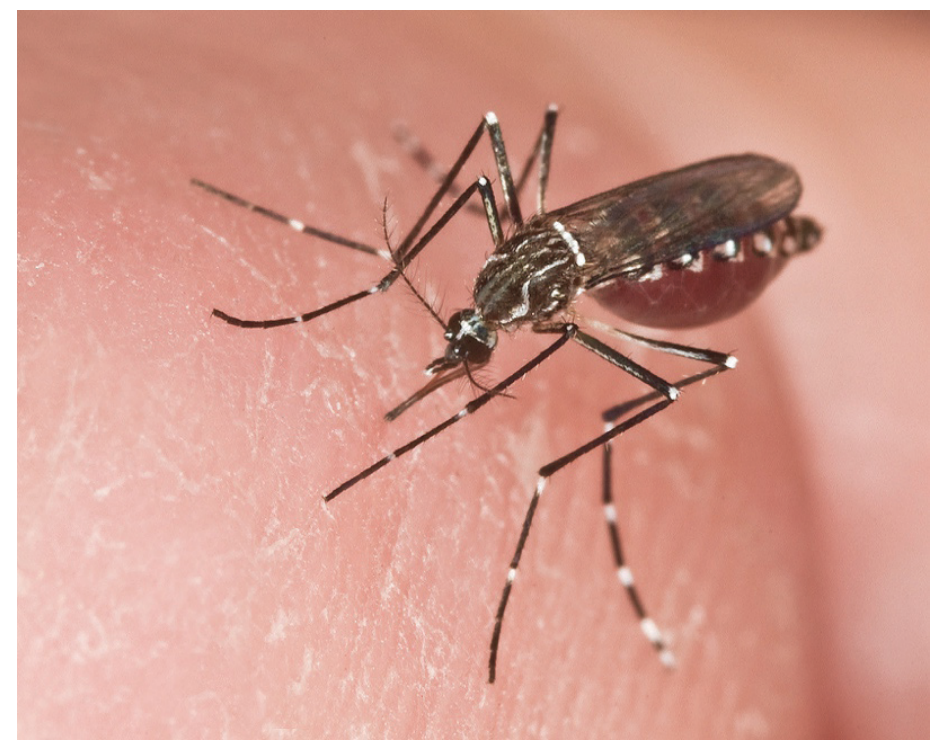

Figure 1. Adult female Aedes aegypti mosquito.

Credits: J. Newman, UF/IFAS

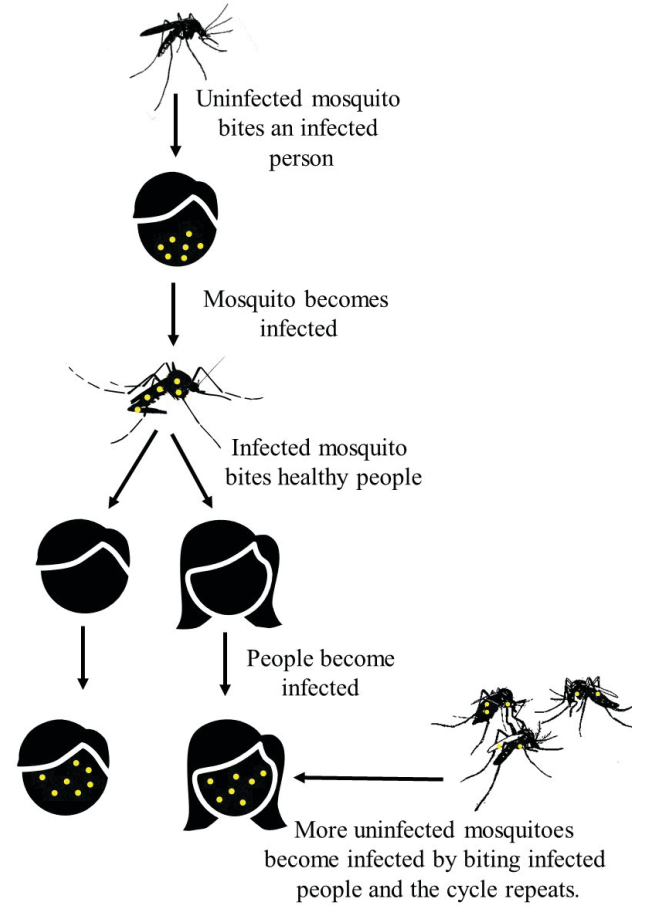

Figure 2. Transmission of mosquito-borne pathogens to people. Credits: A. Romero-Weaver, UF/IFAS

Effective vaccines are not available for most of the diseases caused by Ae. aegypti-transmitted viruses, so disease prevention currently relies on controlling Ae. aegypti mosquito populations. Because Ae. aegypti spend their immature, aquatic life stages (Figure 3 ) in water-filled containers like buckets, jars, and tires (Figure 4), source reduction (removing the containers that mosquitoes may inhabit during their immature, aquatic stages) is essential to Ae. aegypti control. Insecticides are also used to target flying adult mosquitoes. However, these control methods are not perfect. It can be hard to find all the containers inhabited by immature Ae. aegypti mosquitoes to treat with larvicides. Also, over-use and misuse of insecticides like pyrethroids have led to many mosquito populations, including those in Florida, becoming resistant to insecticides. The limited success of conventional control strategies such as source reduction and insecticides against Ae. aegypti has led scientists to search for new tools to target these mosquitoes and reduce the impact of the viruses they spread. One of these new tools is GM mosquitoes.

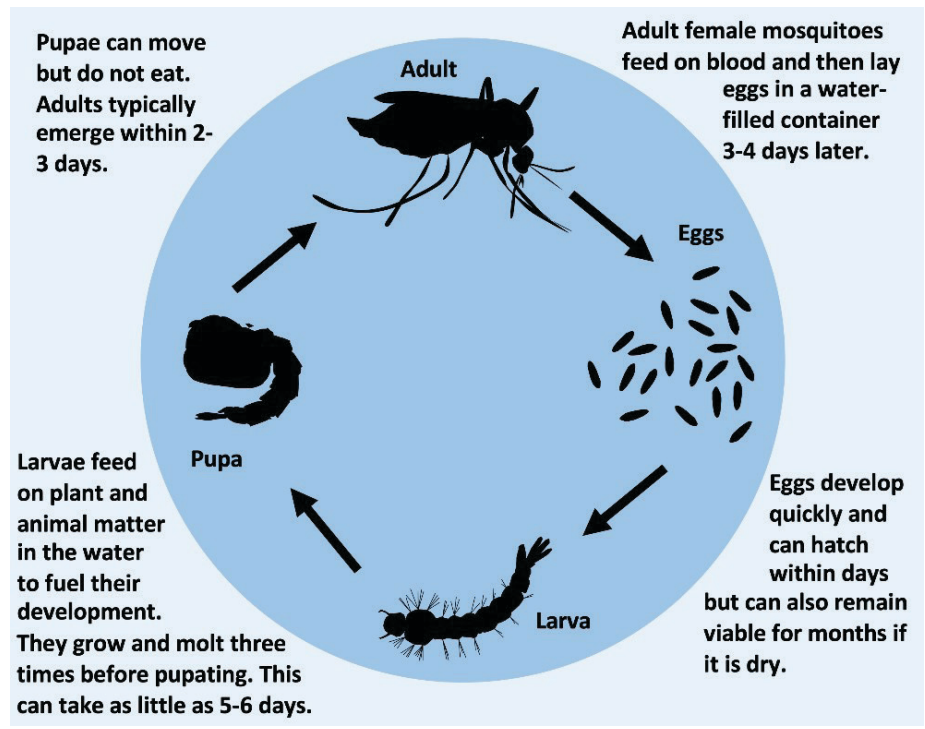

Figure 3. Aedes aegypti mosquito life cycle. Credits: E. P. Caragata, UF/IFAS
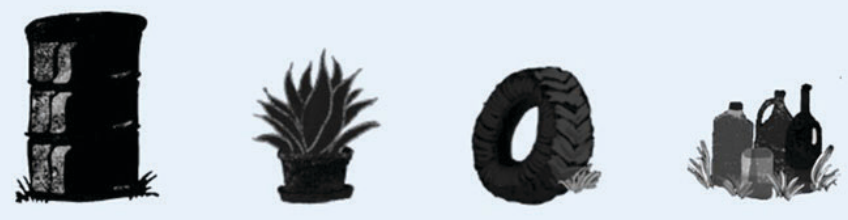

Figure 4. Immature Aedes aegypti mosquitoes are often found in a water collected in containers like used oil drums, flower pots, used tires, and open bottles.

Credits: I. Bargielowski, UC Davis

\section{Mosquito Control Using GM Mosquitoes}

There are two basic approaches to mosquito control using GM mosquitoes. These are known as population suppression and population replacement. Both approaches involve releasing GM mosquitoes into nature. Population suppression is the approach being used in the Oxitec project in Florida. The goal of this approach is to reduce the number 
of mosquitoes in an area over time, which will lower the risk of pathogen transmission. GM mosquitoes used for population suppression are typically male mosquitoes modified so that the survival of the next generation of mosquitoes is reduced. The GM males are released into nature where they mate with wild female mosquitoes. The modification is inherited by their offspring, which might have a greatly reduced hatch rate, or they might die before they develop into adults, depending on the function of the modified gene. As a result of these changes to immature mosquito survival, the population size decreases.

In contrast, the goal of population replacement is not to eliminate mosquitoes from nature, but to replace a wild mosquito population with a modified population that is resistant to infection with important pathogens. Population replacement also involves the release of GM mosquitoes, which pass their pathogen resistance gene to their offspring, and over time mosquitoes in that region become less capable of spreading the pathogen. As with population suppression, there are many genes that can be modified. For instance, genes that produce large quantities of a protein that destroys a specific pathogen can be inserted into the mosquito genome. Alternatively, genes that pathogens need to infect mosquitoes or that promote pathogen replication can be deleted from the mosquito genome. If these GM mosquitoes encounter that pathogen in nature, the modification would make them less likely to become infected with it or spread it between human hosts.

\section{Frequently Asked Questions about Genetically Modified (GM) Mosquitoes}

\section{General Questions}

\section{What is a GM mosquito?}

GM mosquitoes have genomes (genome - the complete genetic material of an organism) that have been altered to make them into effective tools for controlling the spread of mosquito-transmitted pathogens. There are two main control approaches that use GM mosquitoes; population suppression and population replacement (see above), and many different genes that can be modified to reduce mosquito population size or make mosquitoes more resistant to key pathogens.

\section{How are GM mosquitoes developed?}

GM mosquitoes are produced using a technique called embryonic microinjection. This involves injecting freshly laid mosquito eggs with a very small needle containing engineered DNA often called a transgene. This engineered DNA or transgene integrates into the mosquito genome and specifically activates or inactivates a target gene.

\section{The Oxitec and Florida Keys Mosquito Control District Pilot Projects}

\section{What is the goal of the pilot projects?}

The goal is to use GM Ae. aegypti releases as population suppression to reduce the overall number of $A e$. aegypti mosquitoes in the release areas in the Florida Keys. Aedes aegypti, commonly known as the dengue or yellow fever mosquito, is the mosquito species most responsible for transmitting dengue, chikungunya, yellow fever and Zika viruses to humans. It can also transmit dog heartworm to pets. By extension, this would be expected to also impact risk of Ae. aegypti transmitted diseases in the area as well.

\section{What type of GM mosquitoes are being used?}

The mosquitoes being used in the pilot project in the Florida Keys are Oxitec's Friendly ${ }^{\mathrm{TM}}$ GM strain OX5034 of Ae. aegypti mosquitoes. Female Friendly ${ }^{\mathrm{TM}}$ Ae. aegyptimosquitoes die before reaching adulthood, which is when they are capable of biting and spreading pathogens. Adult male Friendly ${ }^{\mathrm{TM}}$ Ae. aegypti mosquitoes, like non-GM male mosquitoes, do not bite and survive to mate with wild non-GM females.

\section{What genetic modifications have been made to the Oxitec Friendly ${ }^{\mathrm{TM}}$ Ae. aegypti GM mosquitoes?}

Two genes have been introduced into the genomes of these GM mosquitoes. The first gene produces a color marker that helps scientists tell the Oxitec Friendly ${ }^{\mathrm{TM}}$ Ae. aegypti mosquitoes apart from wild non-GM mosquitoes. The second gene produces a self-limiting factor, which stops the production of proteins that are essential for mosquito survival, but only in female mosquitoes. This means that only male Oxitec Friendly ${ }^{\mathrm{TM}} A e$. aegypti GM mosquitoes (which do not bite or take blood meals) will survive to adulthood.

\section{What will happen during the projects?}

Researchers and Florida Keys Mosquito Control District personnel will release many male Oxitec Friendly ${ }^{\mathrm{TM}} A e$. aegypti mosquitoes. These will find and mate with wild non-GM female mosquitoes. After mating, the wild female mosquitoes will lay eggs. Immature mosquitoes will hatch 
from the eggs, but because of the self-limiting factor inherited from the Oxitec Friendly ${ }^{\mathrm{TM}}$ Ae. aegypti GM male parental mosquitoes, only male offspring mosquitoes will survive to adulthood. The absence of female offspring mosquitoes in the population will quickly lead to a large
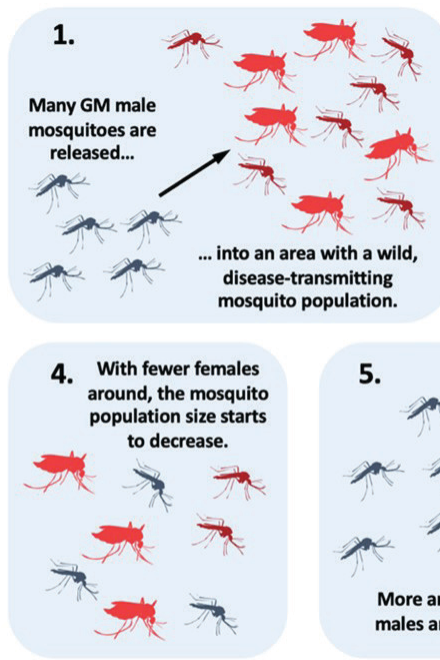

Key:

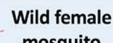
mosquito

Figure 5. How genetically modified mosquito (GM) releases reduce mosquito numbers and mosquito-transmitted disease incidence. Credits: E. P. Caragata, UF/IFAS

drop in the number of Ae. aegypti mosquitoes in the area (Figure 5).

\section{Has the Oxitec technology been tested elsewhere?}

Yes. Oxitec have been testing their GM mosquitoes in other countries, including Brazil (Carvalho et al. 2015), the Cayman Islands (Harris et al. 2012), and Panama for the past ten years. Those experiments have all showed greater than $90 \%$ reduction in the local Ae. aegypti population, meaning the technology was effective.

\section{Impact on Human Health}

\section{Can bites from the released GM Ae. aegypti mosqui- toes make people sick?}

No. Only adult male GM mosquitoes will be released. Male mosquitoes are not capable of biting people or animals as they lack mouthparts capable of piercing skin. Instead, they feed on nectar and other sources of sugar and may even play a role in pollination.

\section{Will the use of GM Ae. aegypti mosquitoes reduce my risk of contracting a mosquito-transmitted disease?}

This is the desired outcome of the project. Mosquitoes can transmit disease-causing pathogens when they bite.
Reducing the number of mosquitoes should mean that people in the release area are bitten less often, which will reduce their risk of mosquito-borne disease exposure. However, only Ae. aegypti mosquitoes are being targeted by this project. There are still other mosquito species in the area that will be unaffected, and the risk associated with the pathogens they transmit, such as West Nile virus, will remain unchanged.

\section{Impact on the Environment}

\section{Is the Ae. aegypti mosquito native to Florida?}

No. Aedes aegypti is not a natural part of the food web in Florida.It is a highly invasive mosquito that was introduced to the United States several hundred years ago during the slave trade. It is now found across Florida and in other parts of the country, including Texas, Arizona, Nevada, and California.

\section{Will the release of Oxitec's Friendly ${ }^{\mathrm{TM}}$ Ae. aegypti mosquitoes disturb the ecosystem?}

This is not very likely. They are not native to Florida. Additionally, no animal relies solely on Ae. aegypti mosquitoes as a food source. Some animals (like bats, frogs, dragonflies, and small fish) may feed on mosquitoes, but they also feed on many other insects, too. So, if there are fewer Ae. aegypti mosquitoes present in an area, animals will eat other insects.

\section{Will Oxitec'sFriendly ${ }^{\mathrm{TM}}$ Ae. aegypti mosquito stay in nature forever?}

No. This technology is designed to be self-limiting, which means that the mosquitoes that Oxitec releases into nature will gradually die off. This is expected to occur a few months after Oxitec and the Florida Keys Mosquito Control District stop releasing mosquitoes.

\section{Will the use of Oxitec's Friendly ${ }^{\mathrm{TM}}$ Ae. aegypti mosqui- toes reduce pesticide use?}

Potentially, if the experiments are successful. In trials conducted in Brazil and the Cayman Islands, the Oxitec technology reduced the local Ae. aegypti mosquito population size by more than $90 \%$. If this success is reproduced in the Florida Keys, it could reduce that area's reliance on the release of chemical pesticides, which is currently 
a common control strategy. Scientists favor integrated mosquito management (IMM) solutions for mosquito control, which means using multiple tools together to give us the best chance of eliminating mosquito populations.

\section{Is it dangerous to introduce genetic mutations into nature?}

Changes to DNA, including genetic mutations, are a regular and natural occurrence in all organisms including animals and humans. In fact, there are often genetic differences that arise between populations of the same mosquito species when they live in different areas. The GM mosquitoes in this project do have two genes introduced into their genomes. There is no existing pathway for any of the engineered genes integrated in the genome of the GM Ae. aegypti to be transmitted to other organisms in a timescale relevant to the period of the trial Additionally, the fact that the Oxitec mosquitoes are self-limiting will further minimize the risk that this type of transfer occurs.

\section{What can I do to help ensure that field trials involving GM mosquitoes are performed in a way that has minimal impact on the environment and provides maximum benefit to those affected by mosquito- transmitted diseases?}

You can make your voice heard in public forums and advocate that robust environmental studies and risk analyses are performed for these projects. You can recommend that stakeholders, including community groups, are consulted throughout the planning and experimentation processes, and that the groups undertaking the research share their data and conclusions. You can also stay informed about the project, so that you can effectively communicate the science behind these projects to interested members of your community. All of these are essential parts of testing new mosquito control technologies.

\section{Additional information}

- Oxitec's website about the pilot projects in Florida: https://www.oxitec.com/florida

- The Centers for Disease Control and Prevention's website about genetically modified mosquitoes: https://www. cdc.gov/mosquitoes/mosquito-control/community/sit/ genetically-modified-mosquitoes.html
- The World Health Organization's position on genetically modified mosquitoes: https://www.who.int/ publications/i/item/9789240013155

- A recent literature review article on genetically modified mosquitoes: https://doi.org/10.1016/j.pt.2018.02.003

\section{References}

Carvalho, D. O., A. R. McKemey, L. Garziera, R. Lacroix, C. A. Donnelly, L. Alphey, A. Malavasi, and M. L. Capurro. 2015. "Suppression of a Field Population of Aedes aegypti in Brazil by Sustained Release of Transgenic Male Mosquitoes." PLoS Negl Trop Dis. 9(7): e0003864. doi: 10.1371/journal.pntd.0003864. PMID: 26135160; PMCID: PMC4489809.

Florida Department of Health (FDOH). 2021. Florida Arbovirus Surveillance Week 53: December 27, 2020-January 2, 2021. 2020 Week 53 Arbovirus Surveillance Report. (Accessed January 6, 2021)

Harris, A. F., A. R. McKemey, D. Nimmo, Z. Curtis, I. Black, S. A. Morgan, M. N. Oviedo, et al. 2012. "Successful Suppression of a Field Mosquito Population by Sustained Release of Engineered Male Mosquitoes." Nat Biotechnol. 30 (9): 828-30. doi: 10.1038/nbt.2350. PMID: 22965050.

Kendrick, K., D. Stanek, and C. Blackmore. 2014. Notes from the field: Transmission of chikungunya virus in the continental United States-Florida, 2014. MMWR Morb Mortal Wkly Rep. 63: 1137.

Philip, C., C. G. Novick, and L. F. Novick. 2019. "Local Transmission of Zika Virus in Miami-Dade County." J Public Health Manag Pract 25:277-287.

Rey, J. R. 2014. “Dengue in Florida (USA).” Insects 5:991-1000. doi: 10.3390/insects5040991 